Document downloaded from:

http://hdl.handle.net/10251/157200

This paper must be cited as:

Ferrando-Rocher, M.; Valero-Nogueira, A.; Herranz Herruzo, Jl.; Teniente, J. (2019). 60GHz Single-Layer Slot-Array Antenna fed by Groove Gap Waveguide. IEEE Antennas and Wireless Propagation Letters. 18(5):846-850. https://doi.org/10.1109/LAWP.2019.2903475

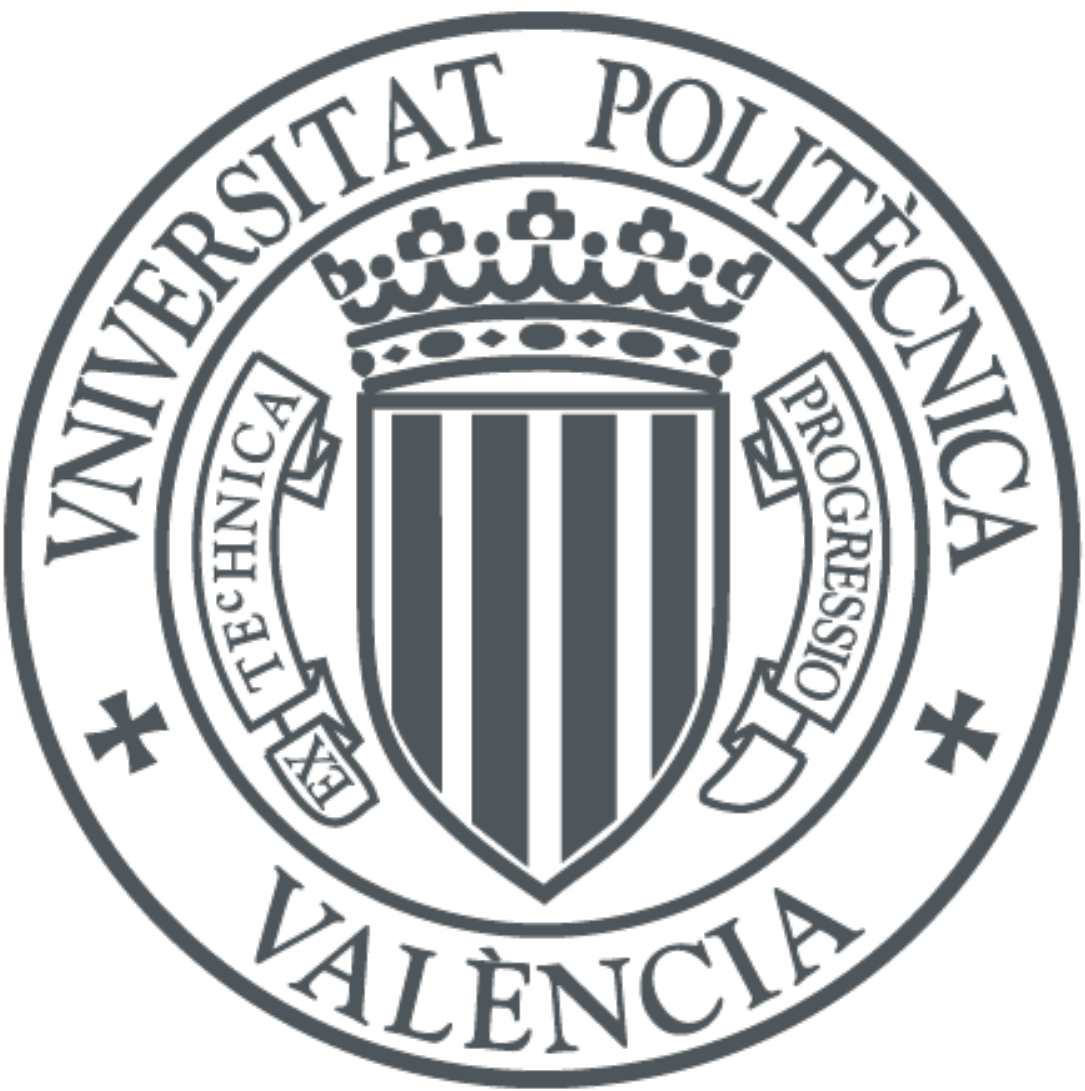

The final publication is available at

https://doi.org/10.1109/LAWP.2019.2903475

Copyright Institute of Electrical and Electronics Engineers

Additional Information 


\title{
60-GHz Single-Layer Slot-Array Antenna fed by Groove Gap Waveguide
}

\author{
Miguel Ferrando-Rocher, Alejandro Valero-Nogueira, Jose I. Herranz-Herruzo, Jorge Teniente
}

\begin{abstract}
A V-band single-layer low-loss slot-array antenna is presented in this paper. Radiating slots are backed by coaxial cavities which are fed through a Groove Gap Waveguide (GGW) E-plane corporate feed network. Cavity resonances are created by shortening nails with respect to the surrounding ones. This fact enables a compact single-layer architecture since coaxial cavities and feeding network can share the same bed of nails. A $16 \times 16$ array is designed, constructed and measured to demonstrate the viability of this concept for high-gain single-layer slot-array antennas. In addition, this solution can be extended to circular polarization by seamlessly adding a polarizer above the slots without changing the feeding network piece. Measurements show a relative bandwidth of $10 \%$ with input reflection coefficient better than $-10 \mathrm{~dB}$ and a mean antenna efficiency above $70 \%$ within the operating frequency band $(57-66 \mathrm{GHz})$.
\end{abstract}

\section{INTRODUCTION}

$60-\mathrm{GHz}$ band is lately receiving increasing attention in wireless world, with both short-range and wider area applications. The development of this band has led to many applications such as high-definition video streaming, high-speed Internet, high-definition multimedia interface or automotive radars [1]. Because of the high propagation losses, one of the key elements for such applications is the antenna, which should be able to provide high directivity and radiation efficiency. Directive antennas for radio links are generally realized by using lens and reflector antennas [2]. However, thin planar array antennas are more attractive for these new applications, due to their lower volume and weight [3].

In this context it is understood the appearance of new full-metal and low-profile solutions. In this regard, hollow waveguide distribution networks are able to feed large arrays with very low losses. Nevertheless, to achieve good electrical contact between the metallic building blocks is a challenging task. To overcome this problem, some planar arrays have been manufactured by using a diffusion bonding technique which is capable of assuring a perfect metallic contact [4]. This fabrication technique, however, is known to be expensive compared to conventional methods [5]. Conversely, a cost-effective alternative technology is the Gap Waveguide (GW) [6]. This waveguiding technology avoids the problem of assuring a good electrical contact thanks to its contactless nature. Notable examples of multilayer GW arrays in this frequency band are presented in [7]-[10]. There exist scarce contributions of GW arrays following a single-layer scheme [11]-[12], but none of them working at $\mathrm{V}$ band.

In this paper, we present a low profile single-layer cavitybacked slot array antenna with high gain (greater than $32 \mathrm{dBi}$ ),

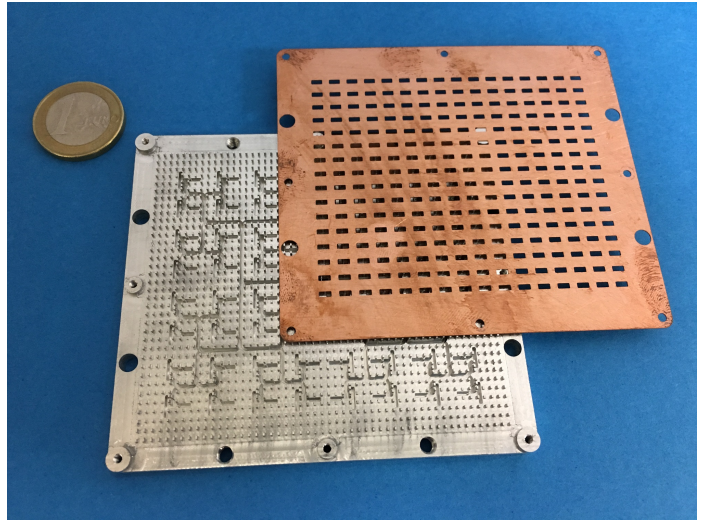

Fig. 1: Manufactured 60-GHz Single-Layer Slot-Array Antenna.

high efficiency (around 70\%), and wide impedance bandwidth (10\%) for the $60-\mathrm{GHz}$ band (see Fig. 1).

\section{BASIC CELL}

The feeding scheme of the basic cell is presented in top and side view in Fig. 2 and Fig. 3, respectively. A similar topology was used in [13] to feed a $4 \times 4$ array in Ka band, though there are some relevant differences as it will be discussed next.

Essentially, the proposed basic cell consists of $2 \times 2$ slots fed by a GGW divider hosted in a bed of nails, where the height of four of them, drawn in purple, is reduced. The height of the rest of the nails is $1.22 \mathrm{~mm}(\lambda / 4$ at $61.5 \mathrm{GHz})$. At the resonance frequency, each GGW branch properly excites the coaxial cavity mode. This fact can be seen in Fig. 2, where the loops of the magnetic field are created around the shortened nails. Interestingly, distance between cavities can be less than $\lambda_{0}$ in this single-layer scheme. In fact, the distance is $0.9 \lambda_{0}$ $\times 0.78 \lambda_{0}$ in the $\mathrm{x}$ and $\mathrm{y}$ direction, respectively.

Unlike the basic cell presented in [13], where each cavity had a coaxial nail with different height, here all the coaxial nails have the same height. So, to excite the four slots with an equal amplitude, an off-centered septum is placed in the 1 to 2 power divider of the basic cell distribution network.

Placing the septum just at the center of the divider (offcentre $=0$ in Fig. 2), the E-field magnitude on the slots is different, as observed in Fig. 4a. However, if the septum is shifted to the right side, the E-field amplitudes are compensated and finally equalized (Fig. 4b). The role of this septum is crucial to distribute the power evenly and to achieve a wide impedance bandwidth (Fig. 5), greater than $14 \%$ (57 to $66 \mathrm{GHz}$ ) for a $\mathrm{S}_{11}$ 


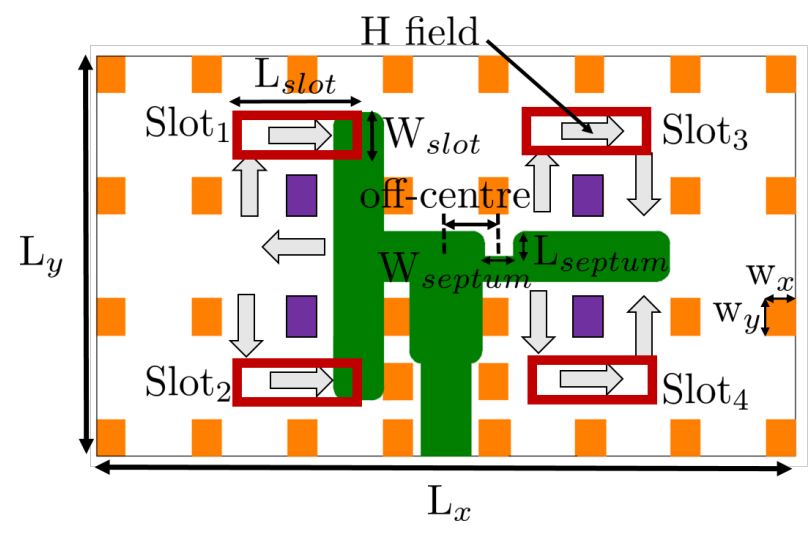

Fig. 2: Basic cell of a $2 \times 2$ subarray fed by GGW. The most representative elements are shown: feeding network (green), shortened-nails (purple), apertures (red) and magnetic field (arrows).

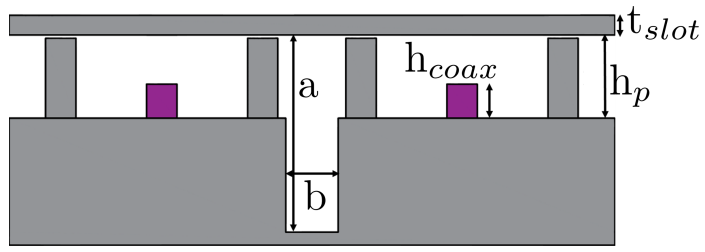

Fig. 3: Side view of the basic cell.

TABLE I: Values of antenna parameters.

\begin{tabular}{|c|c||c|c|}
\hline Parameter & Value $(\mathrm{mm})$ & Parameter & Value $(\mathrm{mm})$ \\
\hline \hline$h_{p}$ & 1.22 & $a$ & 2.96 \\
\hline$h_{\text {coax }}$ & 0.51 & $b$ & 0.8 \\
\hline$w_{x}$ & 0.46 & $L_{x}$ & 9.9 \\
\hline$w_{y}$ & 0.59 & $L_{y}$ & 7.8 \\
\hline$L_{\text {slot }}$ & 2.9 & $W_{\text {slot }}$ & 1.3 \\
\hline$L_{\text {septum }}$ & 0.4 & $W_{\text {septum }}$ & 0.48 \\
\hline of $f_{\text {centre }}$ & 0.85 & $t_{\text {slot }}$ & 0.3 \\
\hline
\end{tabular}

lower than $-15 \mathrm{~dB}$. Notice that such an off-centre septum introduces a small phase shift of few degrees between both branches, though not relevant enough to have an impact on radiation patterns as it will be demonstrated later.

Lastly, after fixing the position of the septum and having previously designed the optimal height of the coaxial nails, a uniform field distribution can be obtained on the four apertures of the subarray.

\section{III. $16 \times 16$ Single-LAYER ARRAY ANTENNA IN V-BAND}

The full array consists on 64 basic cells as the one previously described. The basic cells are fed by a 1 to $64 \mathrm{GGW}$ corporate network. Splitters use step discontinuities to achieve the proper matching, like that used in the basic cell.

Fig. 6 shows a top view of the full structure, where the 1 to 64 corporate-feed distribution network is drawn in green. This compact distribution network allows to place the slots at a distance less than $\lambda_{0}$ in both planes ( $\mathrm{X}$ and $\mathrm{Y}$ ). The nails of the bed are highlighted in orange, having all of them the same height and width. The spacing is larger in the vertical dimension $(1.95 \mathrm{~mm})$ than the horizontal dimension $(1.55 \mathrm{~mm})$, because of the geometry itself of the basic cell. As

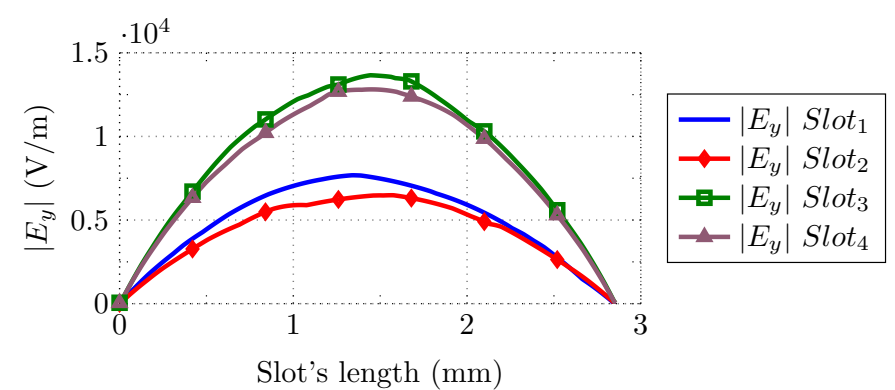

(a)

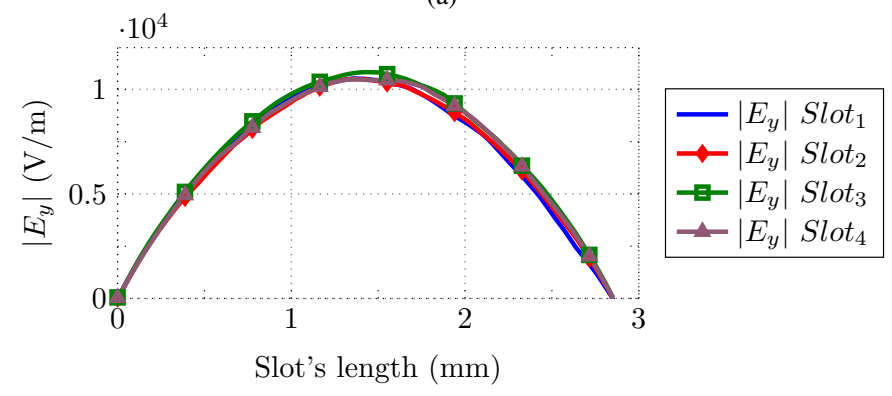

(b)

Fig. 4: Amplitude of the electric field in the apertures using a centered septum and (b) an off-centered septum.

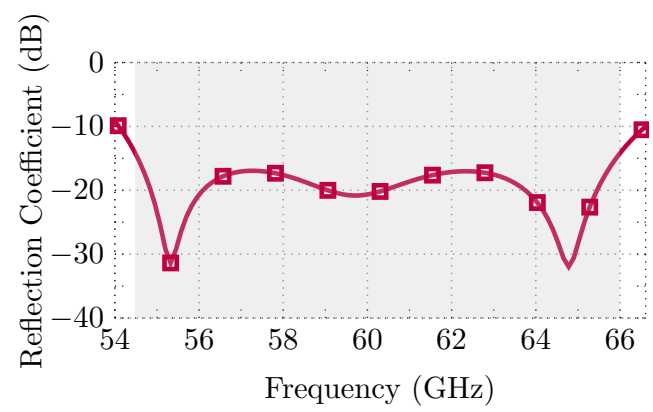

Fig. 5: Simulated reflection coefficient of the basic cell.

for the slots, the ratio $W_{\text {slot }} / L_{\text {slot }}$ was optimized to get the best possible impedance bandwidth. The shortened-nails (purple) are responsible for creating the coaxial cavities. Finally, the apertures (blue) are placed on top. Therefore the antenna is hosting both the distribution network and the cavities exciting the slots in a single layer. All the relevant parameters of the array are included in Table I.

\section{A. Input Port}

The antenna is fed from the backside. Feeding from one side would reduce the complexity of the design but it would have an impact on the radiation pattern. The design of the required transition is not straightforward due to the reduced dimensions of the very input GGW branch.

The optimized transition is shown in Fig. 7. The dimensions of the input WR-15 standard waveguide are $3.7592 \times 1.8796 \mathrm{~mm}^{2}$, whereas the inner dimensions of the GGW are $2.9561 \times 0.8 \mathrm{~mm}^{2}$. Note that the GGW presents one very narrow dimension due to its closeness to the final stages of the corporate feeding network. Such large difference in 


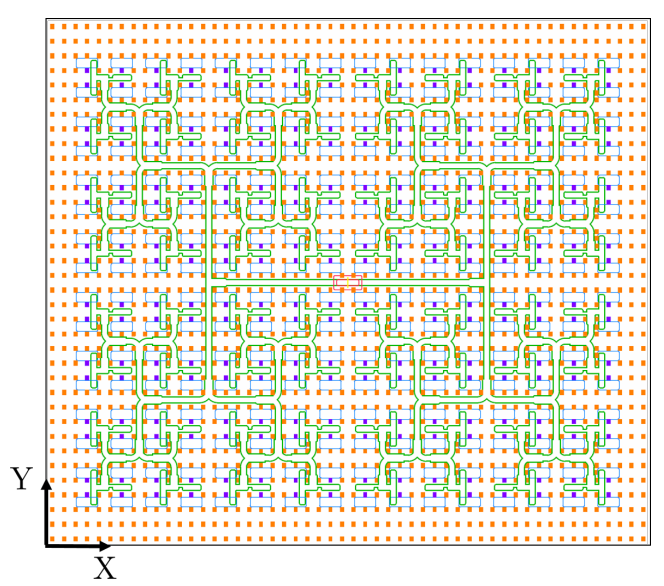

Fig. 6: Top view of the $16 \times 16 \mathrm{~V}$-band linearly-polarized array antenna. The elements that make up the antenna are colored as follows: red (input port), green (corporate-feed network), orange (bed of nails), purple (coaxial nails), blue (apertures).

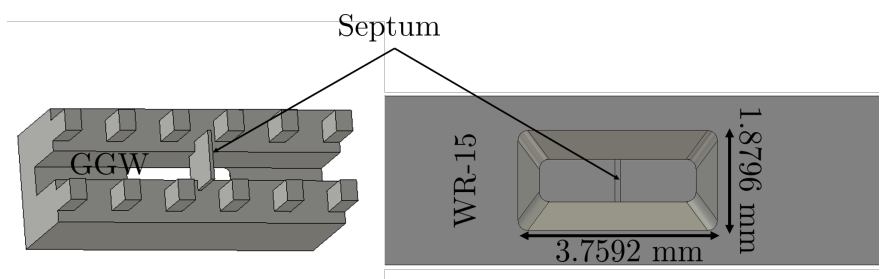

Front View

Back view

(a)

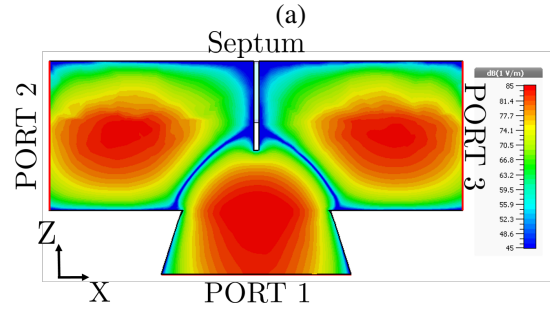

(b)

Fig. 7: (a) (Left) RW-GGW transition without the top plate, where the septum is observed. (Right) Back view of the transition; (b) Magnitude of the electric field within the GGW transition.

dimensions forces us to use a tapered transition between both waveguides, as illustrated in Fig. 7. The transition has been optimized separately from the rest of the antenna to speed up the design process. The optimization process has been mainly focused on tuning the depth $(1.33 \mathrm{~mm})$ and width $(1.16 \mathrm{~mm})$ of the central septum, which have a substantial effect on the return loss.

The main drawback of this solution, however, is that the width must be as thin as possible $(100 \mu \mathrm{m})$ for a proper performance. Hence, this septum would be barely tens of microns wide at higher frequencies, which would complicate fabrication process. In any case, this transition is still a valid and viable solution at $60 \mathrm{GHz}$, as experimental results will show.

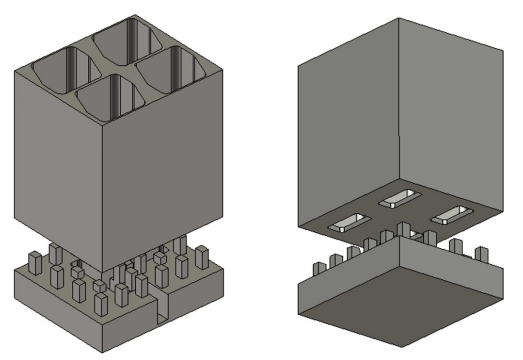

Fig. 8: 3D sketch of the basic cell with the polarizer.

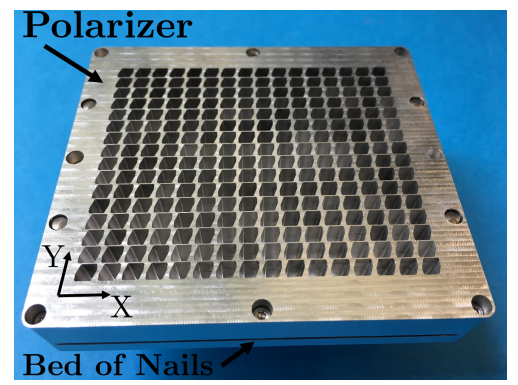

Fig. 9: Manufactured $16 \times 16$ array antenna with polarizer.

TABLE II: Measured antenna parameters of LP array.

\begin{tabular}{cccccc}
\hline Frequency $(\mathrm{GHz})$ & 57 & 59.25 & 61.5 & 63.75 & 66 \\
\hline \hline Directivity $(\mathrm{dBi})$ & 31.22 & 32.64 & 33.16 & 33.26 & 33.35 \\
\hline Gain $(\mathrm{dBi})$ & 30.29 & 31.27 & 32.09 & 31.77 & 31.31 \\
\hline Antenna Efficiency (\%) & 80.72 & 72.95 & 78.16 & 70.96 & 62.52 \\
\hline
\end{tabular}

TABLE III: Measured antenna parameters of CP array.

\begin{tabular}{cccccc}
\hline Frequency $(\mathrm{GHz})$ & 57 & 59.25 & 61.5 & 63.75 & 66 \\
\hline \hline Directivity $(\mathrm{dBi})$ & 32.7 & 33.10 & 33.39 & 33.47 & 33.59 \\
\hline Gain $(\mathrm{dBi})$ & 31.55 & 32.27 & 32.24 & 32.33 & 31.49 \\
\hline Antenna Efficiency $(\%)$ & 76.74 & 82.6 & 76.74 & 76.91 & 61.66 \\
\hline Aperture Efficiency $(\%)$ & 94.77 & 96.17 & 95.43 & 90.46 & 86.76 \\
\hline \hline Axial Ratio (dB) & 3.20 & 2.14 & 1.88 & 3.22 & 3.34 \\
\hline
\end{tabular}

\section{B. Polarizer}

As it is well known, circular polarization $(\mathrm{CP})$ may be very advantageous for certain applications. For that reason, a polarizing layer was designed to seamlessly convert the linear into circular polarization, keeping the same array as a feeder. In [14] a metal cavity mounted on a slot was proposed to achieve a CP antenna. Setting the height and the cross-section of the cavity, two orthogonal modes with equal amplitude but a phase-shift of $90^{\circ}$ can be obtained. Here the same concept is used to transform linear polarization (LP) into CP.

Feeding layer piece is kept the same as in the LP antenna. Therefore, above the bed of nails, new radiators are located. The height of the metal cavity is key in order to get a good polarization purity. The cross-section could be designed in different ways, always under the premise of getting a phase shift of $90^{\circ}$ between two orthogonal modes and equal amplitude. Here, a chamfered square shape is used. In Fig. 8 two views of the basic cell are shown. In the new piece, the slots are integrated directly with the polarizer. The manufactured polarizer is shown in Fig. 9. 

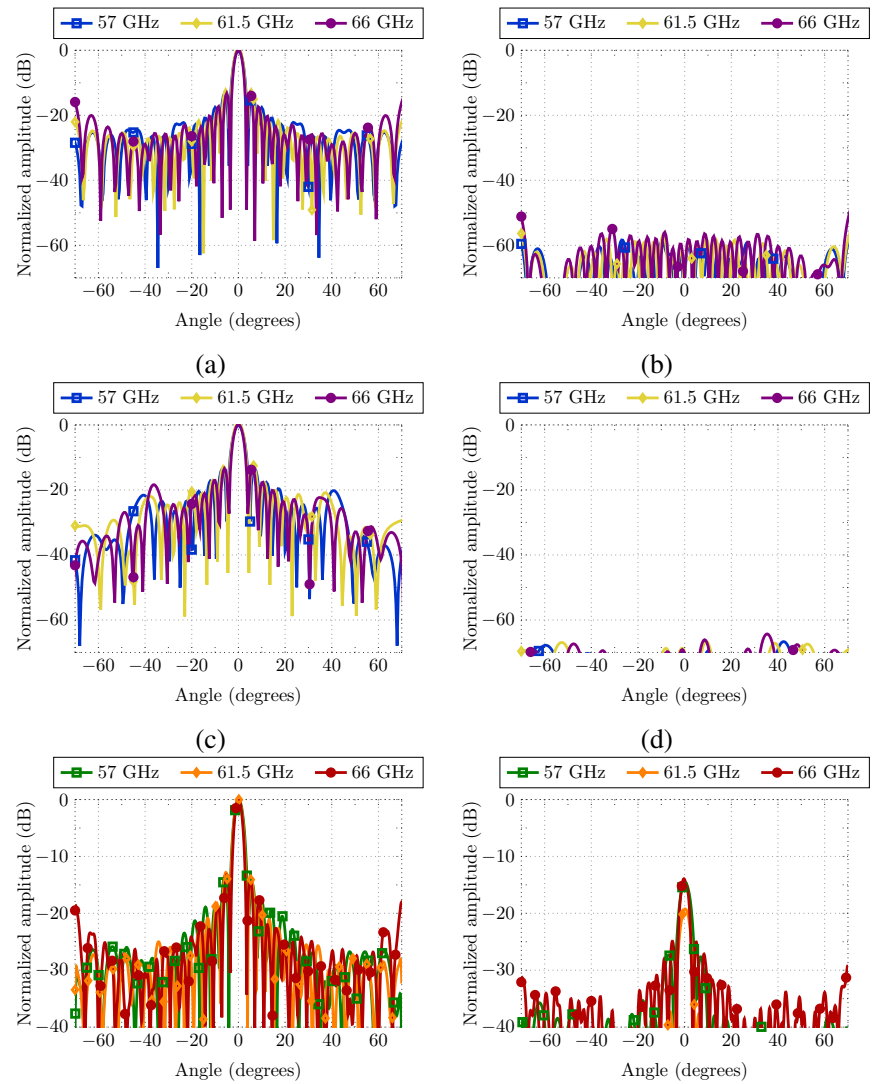

(e)

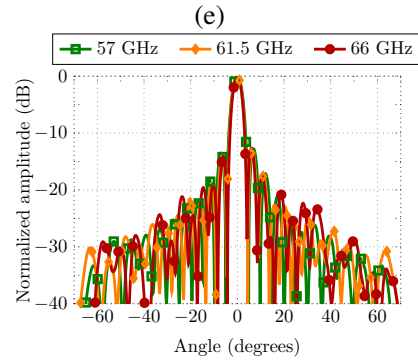

(g)

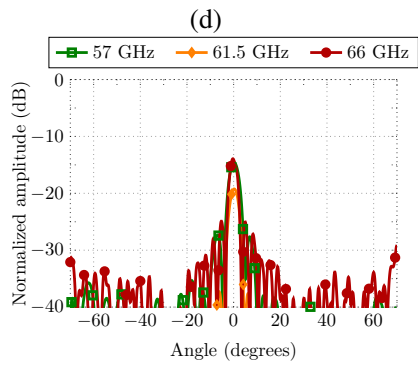

(f)

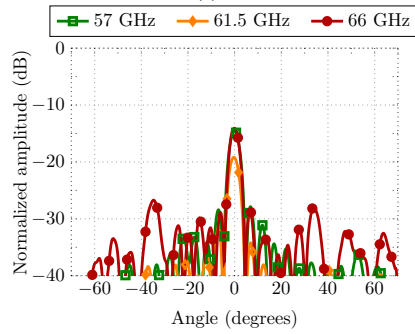

(h)

Fig. 10: Normalized measured radiation patterns for several frequencies for LP antenna: (a) Copolar XZ-plane, (b) Crosspolar XZ-plane, (c) Copolar YZ-plane, (d) Crosspolar YZ-plane. Normalized measured radiation patterns for several frequencies for $\mathrm{CP}$ antenna: (e) Copolar XZ-plane, (f) Crosspolar XZ-plane, (g) Copolar YZ-plane, (h) Cross-polar YZ-plane.

\section{EXPERIMENTAL RESULTS}

Both antennas (LP and CP) were manufactured in-house using a CNC milling machine. It is relevant to note that both were designed taking into account the inner rounded corners with a radius of $200 \mu \mathrm{m}$ due to restrictions on the dimensions of the milling tools. The measured radiation patterns along both main cuts are shown in Fig 10. The large bandwidth of the distribution network enables a great pattern stability within the entire bandwidth, from $57 \mathrm{GHz}$ to $66 \mathrm{GHz}$. However, a slight asymmetry in the radiation patterns is observed due to some uncertainty in the alignment of the antenna in the anechoic chamber. Tables II and III show the measured directivity, gain

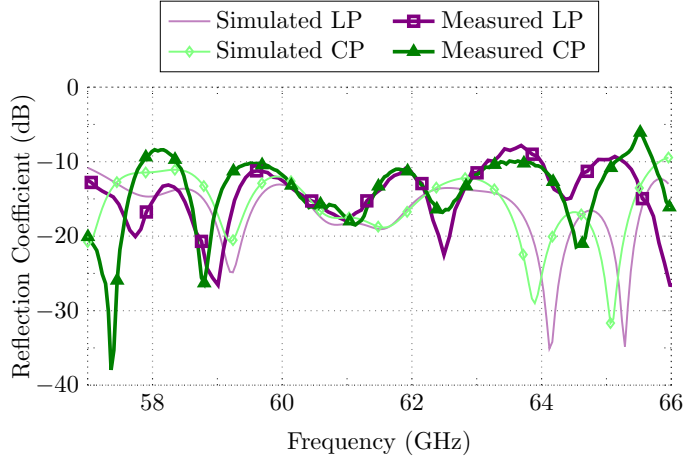

Fig. 11: Measured reflection coefficients.

TABLE IV: Performance comparison between proposed and reported $16 \times 1660-\mathrm{GHz}$ planar antenna arrays in $\mathrm{GW}$.

\begin{tabular}{cccccc}
\hline GW Arrays & BW & Max. Gain & Mean Eff. & Pol. & Pieces \\
\hline \hline Present Work & $14 \%$ & $32.3 \mathrm{dBi}$ & $75 \%$ & LP/CP & 2 \\
\hline Ref. [8] & $16 \%$ & $32.5 \mathrm{dBi}$ & $70 \%$ & LP & 3 \\
\hline Ref. [9] & $17 \%$ & $30.5 \mathrm{dBi}$ & $55 \%$ & LP & 4 \\
\hline Ref. [10] & $18 \%$ & $32.5 \mathrm{dBi}$ & $80 \%$ & LP & 3 \\
\hline
\end{tabular}

and antenna efficiency of both prototypes. In addition, Table III includes the axial ratio of the $\mathrm{CP}$ antenna. A directivity higher than $32 \mathrm{dBi}$ has been obtained with an average antenna efficiency greater than $70 \%$. In addition, the axial ratio shows an acceptable polarization purity, although at some frequency points a value higher than $3 \mathrm{~dB}$ is measured. This is very likely due to the sensitivity of the axial ratio to manufacturing inaccuracies and it is open for further improvement.

Regarding the $S_{11}$, a comparison between simulation and measurement is presented in Fig. 11. A good agreement is observed between both curves for the lower part of the band. A greater deviation is noticed for the upper part, though. However, the $S_{11}$ remains close to the $-10 \mathrm{~dB}$ target over the entire band. Also, after manufacture and measurement, a metrology study was made. Some discrepancies between designed and manufactured model were observed. A deviation of $120 \mu \mathrm{m}$ on the depth of the groove was found. Groove depth governs the waveguide propagation coefficient and it is probably one of the main reasons for the reflection coefficient discrepancy. Finally, in Table IV present work is compared with other recent contributions on GW arrays in V-band.

\section{Conclusions}

A 60-GHz Single-Layer Slot-Array Antenna fed by GGW is presented. Measurements verify the potential of the $\mathrm{GW}$ coaxial cavities to feed single-layer high-gain slot arrays in this band. One distinctive feature of this antenna is the possibility to alternate the lids for linear or circular polarization, using the same bed of nails.

\section{ACKNOWLEDGEMENTS}

This work has been supported by the Spanish Ministry of Economy and Competitiveness (Ministerio de Economía y Competitividad) under the project TEC2016-79700-C2-1-R. Also, the authors want to acknowledge Yebes Astronomical Observatory for experimental validation of the antenna. 


\section{REFERENCES}

[1] F. F. Manzillo, M. Śmierzchalski, L. Le Coq, M. Ettorre, J. Aurinsalo, K. T. Kautio, M. S. Lahti, A. E. Lamminen, J. Säily, and R. Sauleau, "A wide-angle scanning switched-beam antenna system in ltcc technology with high beam crossing levels for v-band communications," IEEE Transactions on Antennas and Propagation, 2018.

[2] E. Alfonso and P.-S. Kildal, "Parabolic cylindrical reflector antenna at $60 \mathrm{GHz}$ with line feed in gap waveguide technology," in Antennas and Propagation (EuCAP), 2013 7th European Conference on. IEEE, 2013, pp. $319-323$.

[3] A. Vosoogh, P.-S. Kildal, and V. Vassilev, "Wideband and High-Gain Corporate-Fed Gap Waveguide Slot Array Antenna With ETSI Class II Radiation Pattern in V-Band," IEEE Transactions on Antennas and Propagation, vol. 65, no. 4, pp. 1823-1831, 2017.

[4] M. Zhang, K. Toyosaki, J. Hirokawa, M. Ando, T. Taniguchi, and M. Noda, "A 60-ghz band compact-range gigabit wireless access system using large array antennas," IEEE Transactions on Antennas and Propagation, vol. 63, no. 8, pp. 3432-3440, 2015.

[5] S. M. Lewis, E. A. Nanni, and R. J. Temkin, "Direct machining of lowloss $\mathrm{THz}$ waveguide components with an RF choke," IEEE Microwave and Wireless Components Letters, vol. 24, no. 12, pp. 842-844, 2014.

[6] P.-S. Kildal, E. Alfonso, A. Valero-Nogueira, and E. Rajo-Iglesias, "Local metamaterial-based waveguides in gaps between parallel metal plates," IEEE Antennas and Wireless Propagation Letters, vol. 8, pp. 84-87, 2009.

[7] A. Vosoogh and P.-S. Kildal, "Corporate-fed planar 60-GHz slot array made of three unconnected metal layers using AMC pin surface for the gap waveguide," IEEE Antennas and Wireless Propagation Letters, vol. 15, pp. 1935-1938, 2016.

[8] D. Zarifi, A. Farahbakhsh, A. U. Zaman, and P.-S. Kildal, "Design and fabrication of a high-gain $60-\mathrm{GHz}$ corrugated slot antenna array with ridge gap waveguide distribution layer," IEEE Transactions on Antennas and Propagation, vol. 64, no. 7, pp. 2905-2913, 2016.

[9] J. Liu, A. Vosoogh, A. U. Zaman, and J. Yang, "Design and fabrication of a high-gain 60-ghz cavity-backed slot antenna array fed by inverted microstrip gap waveguide," IEEE Transactions on Antennas and Propagation, vol. 65, no. 4, pp. 2117-2122, 2017.

[10] A. Farahbakhsh, D. Zarifi, and A. U. Zaman, "60-GHz Groove Gap Waveguide Based Wideband H-Plane Power Dividers and Transitions: For Use in High-Gain Slot Array Antenna," IEEE Transactions on Microwave Theory and Techniques, vol. 65, no. 11, pp. 4111-4121, 2017.

[11] M. Ferrando-Rocher, J. I. Herranz, A. Valero-Nogueira, and B. Bernardo, "Performance assessment of gap waveguide array antennas: Cnc milling vs. 3d printing," IEEE Antennas and Wireless Propagation Letters, 2018.

[12] M. Ferrando-Rocher, J. I. Herranz-Herruzo, A. Valero-Nogueira, and A. Vila-Jiménez, "Single-layer circularly-polarized ka-band antenna using gap waveguide technology," IEEE Transactions on Antennas and Propagation, 2018.

[13] A. J. Sáez, A. Valero-Nogueira, J. I. Herranz, and B. Bernardo, "Singlelayer cavity-backed slot array fed by groove gap waveguide," IEEE Antennas and Wireless Propagation Letters, vol. 15, pp. 1402-1405, 2016.

[14] D. Dogan and C. B. Top, "Circularly polarized ka-band waveguide slot array with low sidelobes," in Antennas and Propagation (EUCAP), 2012 6th European Conference on. IEEE, 2012, pp. 1105-1109. 